\title{
The MBARI-WEC: a power source for ocean sensing
}

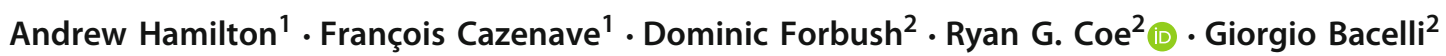

Received: 22 October 2020 / Accepted: 27 April 2021 / Published online: 24 May 2021

(c) The Author(s). This is a U.S. government work and not under copyright protection in the U.S.; foreign copyright protection may apply 2021

\begin{abstract}
Interest in wave energy converters to provide autonomous power to various ocean-bound systems, such as autonomous underwater vehicles, sensor systems, and even aquaculture farms, has grown in recent years. The Monterey Bay Aquarium Research Institute has developed and deployed a small two-body point absorber wave energy device suitable to such needs. This paper provides a description of the system to support future open-source access to the device and further the general development of similar wave energy systems. Additionally, to support future control design and system modification efforts, a set of hydrodynamic models are presented and cross-compared. To test the viability of using a linear frequency-domain admittance model for controller tuning, the linear model is compared against four WEC-Sim models of increasing complexity. The linear frequency-domain model is found to be generally adequate for capturing system dynamics, as the model agreement is good and the degree of nonlinearity introduced in the WEC-Sim models is generally less than $2.5 \%$.
\end{abstract}

Keywords Wave energy converter (WEC) · Powering the blue economy (PBE) · Hydrodynamic modeling

\section{Introduction}

While much of the work to date on WECs has been targeted at "utility-scale" devices, which would have capacities on the order of $1 \mathrm{MW}$, in line with wind turbines, there is now increasing interest in smaller devices. Smaller WECs may be used for a more distributed approach (i.e., with a "farm" of many WECs needed to give a MW of generating capacity, as suggested by Falnes and Hals 2012) or for applications where the power needs are lower, such as the so-called blue economy (Cavagnaro et al. 2020; LiVecchi et al. 2019; Copping et al. 2018). Such a device might be used to power remotely

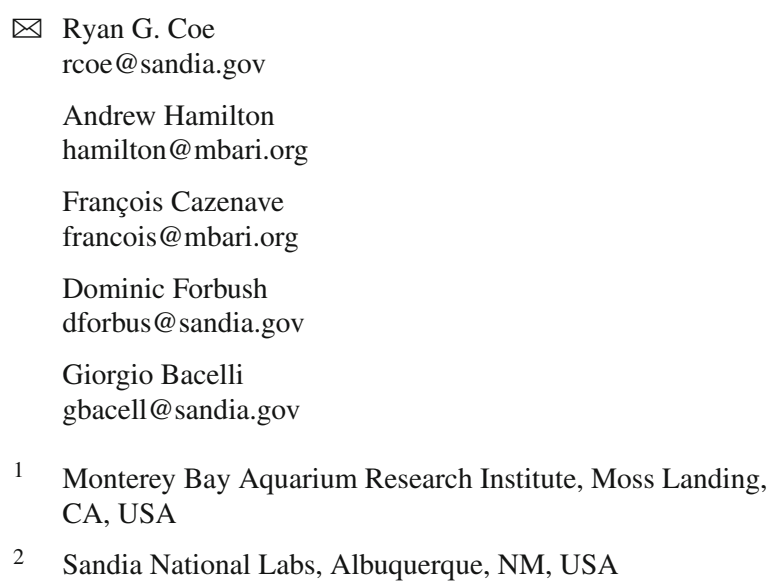

2 Sandia National Labs, Albuquerque, NM, USA

located sensors or charge autonomous vehicles (Green et al. 2019; Driscol et al. 2020; Hagerman 2002).

This paper provides an overview of the Monterey Bay Aquarium Research Institute (MBARI) wave energy converter (WEC) - the device is herein referred to as the "MBARI-WEC." The MBARI-WEC was originally developed in 2009 to provide power to MBARI's various research missions. The project was initially funded by the Defense Advanced Research Projects Agency (DARPA), as a 3-month grant to assess the availability of wave power around the world, and to assess DARPA's previous attempts to generating electrical power from ocean waves. The project has been continued since then, with support from MBARI's main funding source, the David and Lucille Packard Foundation.

First, a detailed description of the system is provided (Sect. 2). Next, to support future control design and system re-design efforts, a series of hydrodynamical models are proposed and cross-compared (Sects. 3-4). These findings are discussed and summarized in Sects. 5 and 6, respectively.

\section{Device description}

The MBARI wave-energy conversion buoy is a two-body point absorber that consists of a surface buoy and submerged heave-plate connected by an electro-hydraulic power take-off 


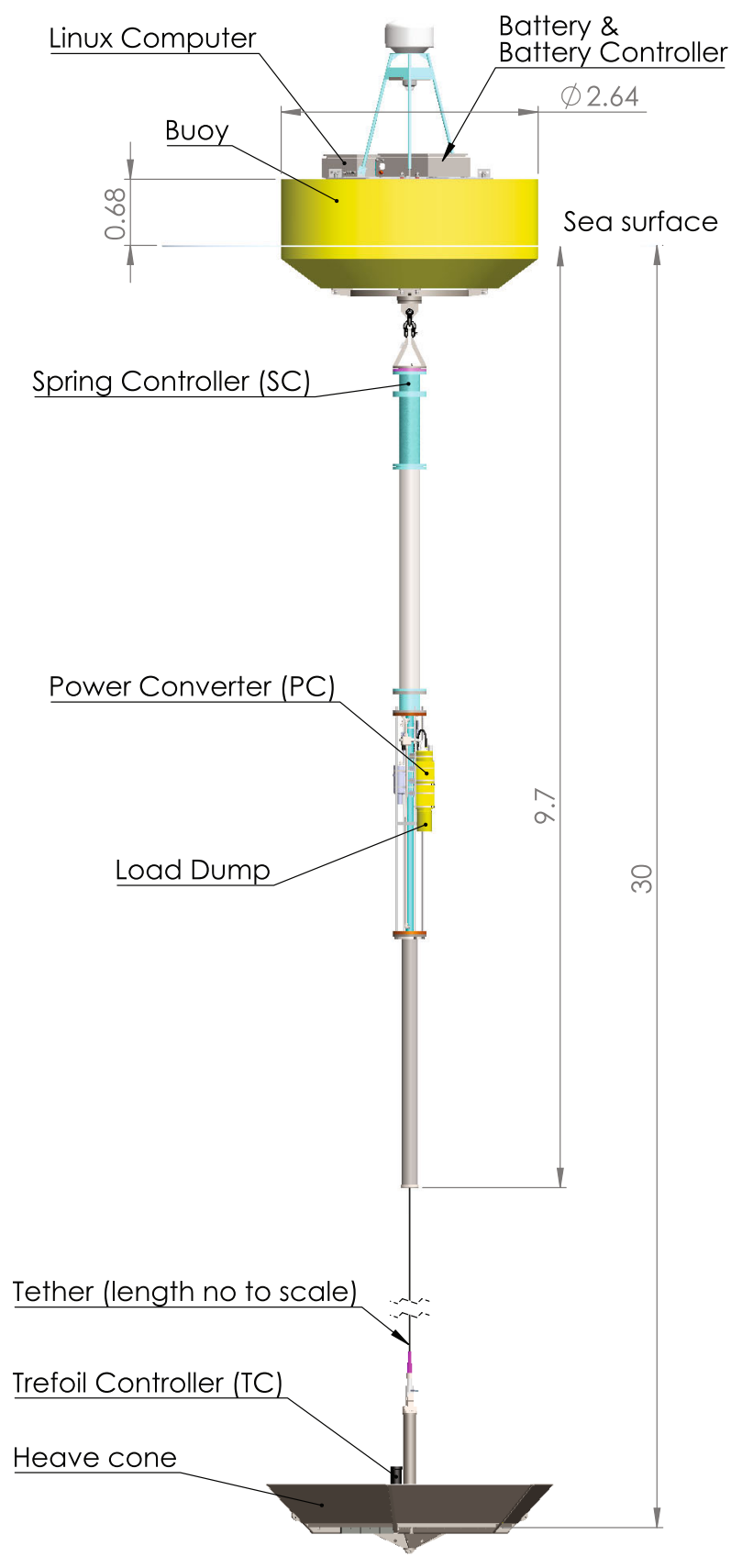

Fig. 1 MBARI-WEC system diagram showing mechanical layout along with electronic subsystem locations. Dimensions are in meters

unit. A layout diagram is shown in Fig. 1 and key parameters for the device are given in Table 1. Power and communications extend from the buoy down to the heave-cone, but do not extend to the sea floor at this time. Typically, the system is deployed with the submerged heave cone anchored to the sea floor with a chain-catenary mooring and a $180 \mathrm{~kg}$ (400 lb) "Dormor" anchor. The usual deployment location is $36.7436 \mathrm{~N},-121.88 \mathrm{E}$ in roughly $80 \mathrm{~m}$ of water (Fig. 2).
The computing and software architecture of the system is distributed, as shown in Fig. 3, using micro-controllers in critical roles and a topside Linux computer to log telemetry, issue commands to the micro-controllers when needed, and provide shore-based control of the system. In particular, there are four micro-controllers throughout the system, serving as a battery controller (BC), a power-converter controller (PC), a pneumatic-spring controller (SC), and a heave-cone trefoil controller (TC). The locations of each of these microcontrollers are shown in Fig. 1. A listing of telemetry signals provided by each micro-controller is presented in Table 6 in the "Appendix".

In each case, these controllers broadcast a stream of data on a Controller Area Network (CAN) bus continuously at up to $50 \mathrm{~Hz}$. The topside Linux computer time-stamps and logs all of this data, and provides a mechanism for data transmission to shore via terrestrial radio or cell-phone modem. Each micro-controller can also accept commands from the Linux computer to execute a function or change behavior. In addition to interfacing with the micro-controllers, the Linux computer logs data from a inertial measurement unit (IMU)/global positioning system (GPS) (Crossbow Nav440) and a SimRad radar system currently being used for incoming wave-detection research.

\subsection{Buoy subassembly}

A layout drawing of the MBARI-WEC buoy is shown in Fig. 4. On board the buoy are two watertight boxes: one contains the battery system and the other contains the buoy control Linux computer and associated hardware. The battery has a capacity of $5 \mathrm{~kW}-\mathrm{Hrs}(17 \mathrm{~A}-\mathrm{Hrs} @ 325 \mathrm{~V})$ and this housing also includes a $325-24 \mathrm{~V}$ power supply that is the main power supply to the buoy controller, a battery control micro-controller which provides power switching, manages over- and under-charge conditions as well as battery balancing, and monitors environmental conditions such as hydrogen levels and ground faults on the $325 \mathrm{~V}$ bus.

The controller box contains a Linux computer, an Ethernet network, a $2.4 \mathrm{Ghz}$ Wi-Fi radio, a $4 \mathrm{G}$ cellular modem, power supplies that generate $12 \mathrm{~V}$ and $5 \mathrm{~V}$ from the incoming $24 \mathrm{~V}$, and a power switching relay that can switch any voltage in this box to external devices as needed. Not shown in Fig. 4 are the antennas for the two radios (Wi-Fi and cellular modem), a light, and an independent satellite location transponder ("SPOT tracker").

\subsection{Heave plate subassembly}

The heave cone (Fig. 5) is located $30 \mathrm{~m}$ below the free surface, within the depth limit for a typical open-water diver. The heave cone is designed to be adaptable and can open and close the center aperture by rotating the clover-shaped "trefoil" 
Table 1 MBARI-WEC key parameters

\begin{tabular}{lll}
\hline Parameter & Value & Notes \\
\hline Total system mass, $m_{t}[\mathrm{~kg}]$ & 2530 & Includes PTO system \\
PTO pneumatic spring const., $K_{\text {spring }}[\mathrm{kN} / \mathrm{m}]$ & 8.9 & Spring not purely linear, see Sect. 2.3 \\
Buoy & & \\
Mass, $m_{1}[\mathrm{~kg}]$ & 1080 & See Fig. 9 \\
Natural period, $T_{1,0}[\mathrm{~s}]$ & 1.4 & Below buoy deck, see 'G' mark in Fig. 4 \\
Center of mass, $z_{1, \operatorname{cog}}[\mathrm{m}]$ & 0.67 & Below buoy deck, see 'B' mark in Fig. 4 \\
Center of buoyancy, $z_{1, \mathrm{cob}}[\mathrm{m}]$ & 0.98 & \\
Metacentric height $[\mathrm{m}]$ & 1.93 & Below buoy deck (when PTO and heave cone are attached) \\
Water line $[\mathrm{m}]$ & 0.80 & \\
Water plane area, $A_{w p}\left[\mathrm{~m}^{2}\right]$ & 5.47 & \\
2nd-moment of area of water plane $\left[\mathrm{m}^{4}\right]$ & 1.37 & \\
Heave cone & & \\
Mass, $m_{2}[\mathrm{~kg}]$ & 815 & See Fig. 9 \\
Natural period, $T_{2,0}[\mathrm{~s}]$ & 7.9 & Below attachment point, see 'G' mark in Fig. 5 \\
Center of mass, $z_{2, \operatorname{cog}}[\mathrm{m}]$ & 1.25 & See Fig. 1 \\
Depth location $[\mathrm{m}]$ & 30 &
\end{tabular}

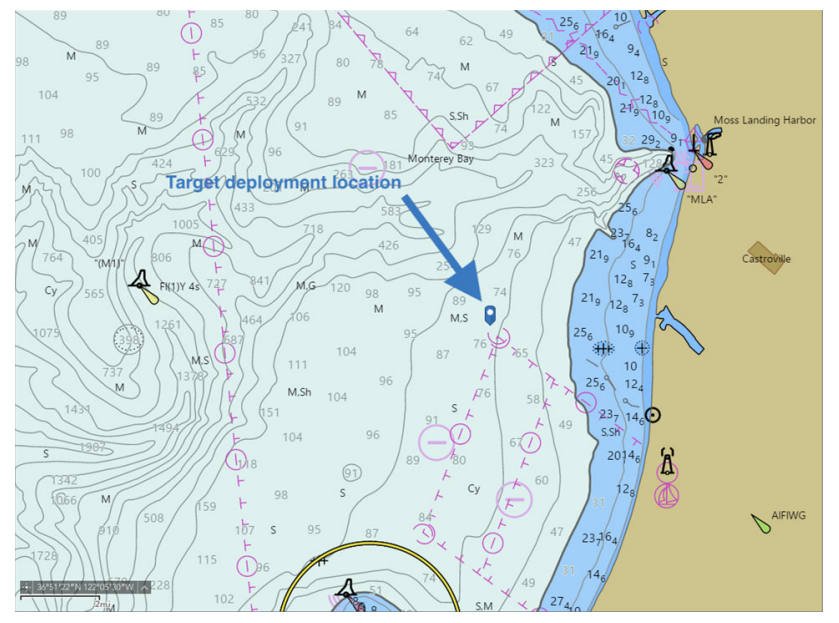

Fig. 2 Monterey Bay bathymetry at deployment location (36.7436N$121.88 \mathrm{E}$ ) shown with blue marker and arrow. Depths shown in meters. (reproduced from https://www.nauticalcharts.noaa.gov/ENCOnline/ enconline.html)

upon command for purposes of storm-survivability. The two configurations of the heave cone have dramatically different added mass values and modestly different drag coefficients. These values have been estimated by potential flow analysis and CFD studies, and have been confirmed in tank tests. The heave cone contains a micro-controller that controls the opening and closing of the doors, and also reports telemetry from a pressure sensor and inexpensive IMU (VectorNav VN-100). The Linux computer monitors PTO extension and opens the doors when larger seas cause excursions beyond $80 \%$ of the total stroke, and closes the doors upon a command from shore. This controller also contains a battery back-up

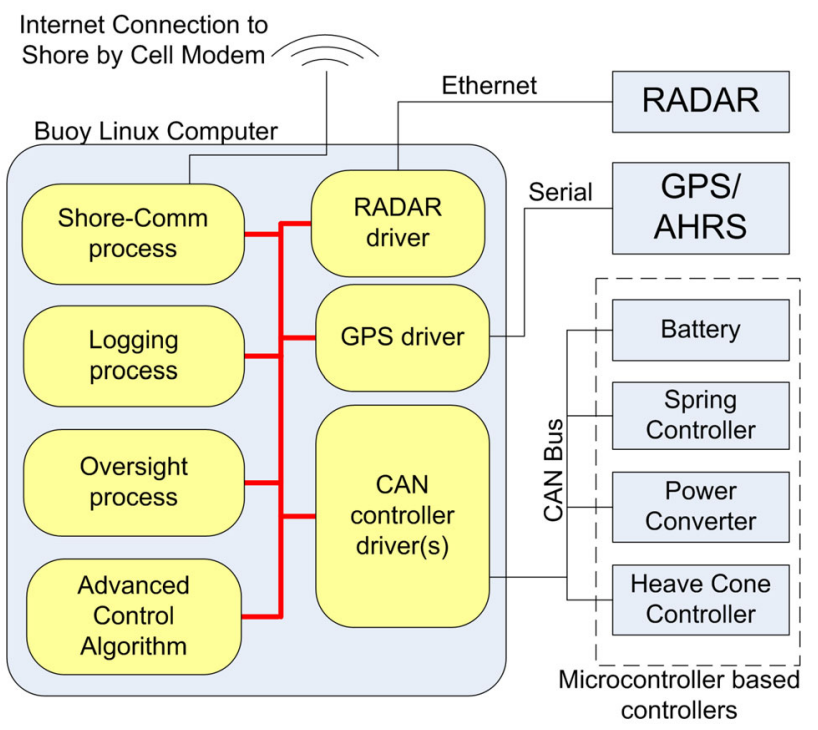

Fig. 3 MBARI-WEC buoy computing and software infrastructure

and fail-safe logic that opens the doors in the case power or communications are lost from the surface. The tether from the PTO to the heave cone contains two larger power conductors and four smaller communications lines. Currently, communication is provided by extending the system CAN bus down the tether and power is transmitted at $48 \mathrm{~V}$.

\subsection{PTO subassembly}

The PTO system consists of an electro-hydraulic system for power capture and a pneumatic spring device that provides 


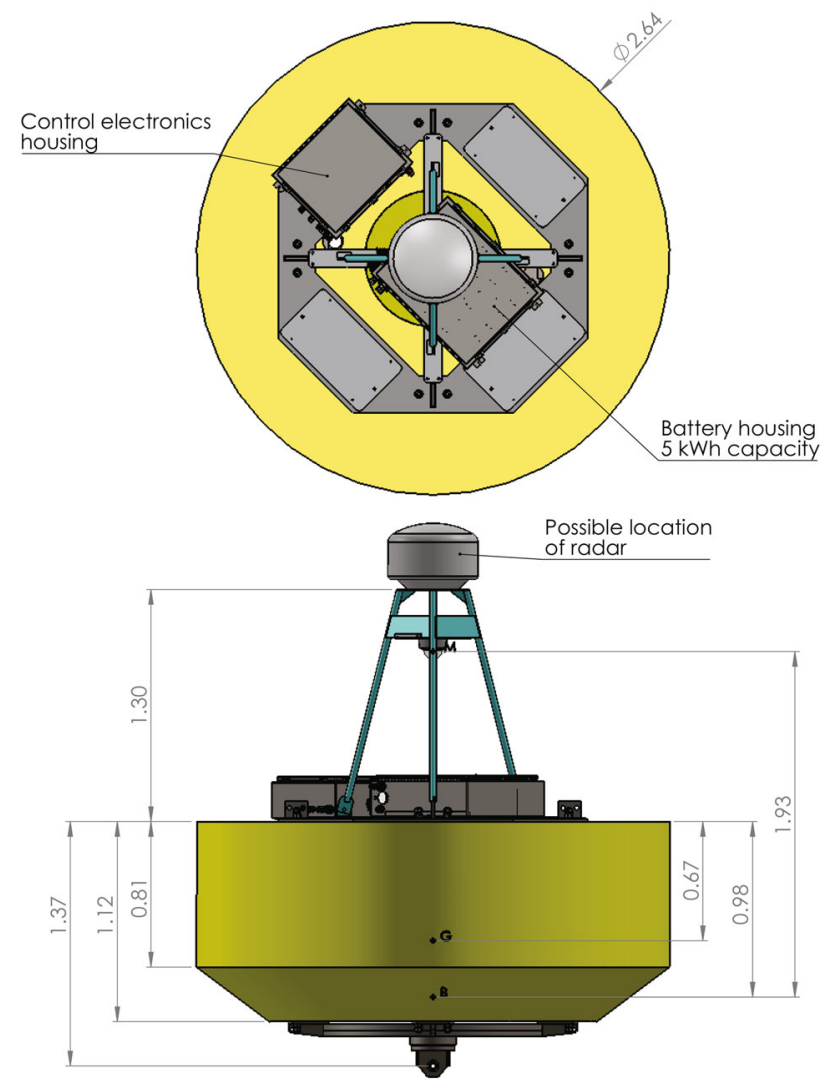

Fig. 4 MBARI-WEC buoy layout drawing with top and side views. Dimensions are in meters

a restoring force (Fig. 6). These two elements (electrohydraulic system and a pneumatic spring) are effectively connected in parallel. The PTO couples the buoy and heave cone via a flexible tether.

The pneumatic spring component provides a restoring force of about $8.9 \mathrm{kN}(2000 \mathrm{lbs})$ per meter of extension. The static weight of the heave-cone therefore extends the PTO approximately half-way in calm seas. The pneumatic spring also contains a micro-controller that provides a variety of features. It is primarily a telemetry device that measures and reports (at $10-50 \mathrm{~Hz}$ ) the spring extension, the upper and lower gas pressures, and the tension between the PTO and
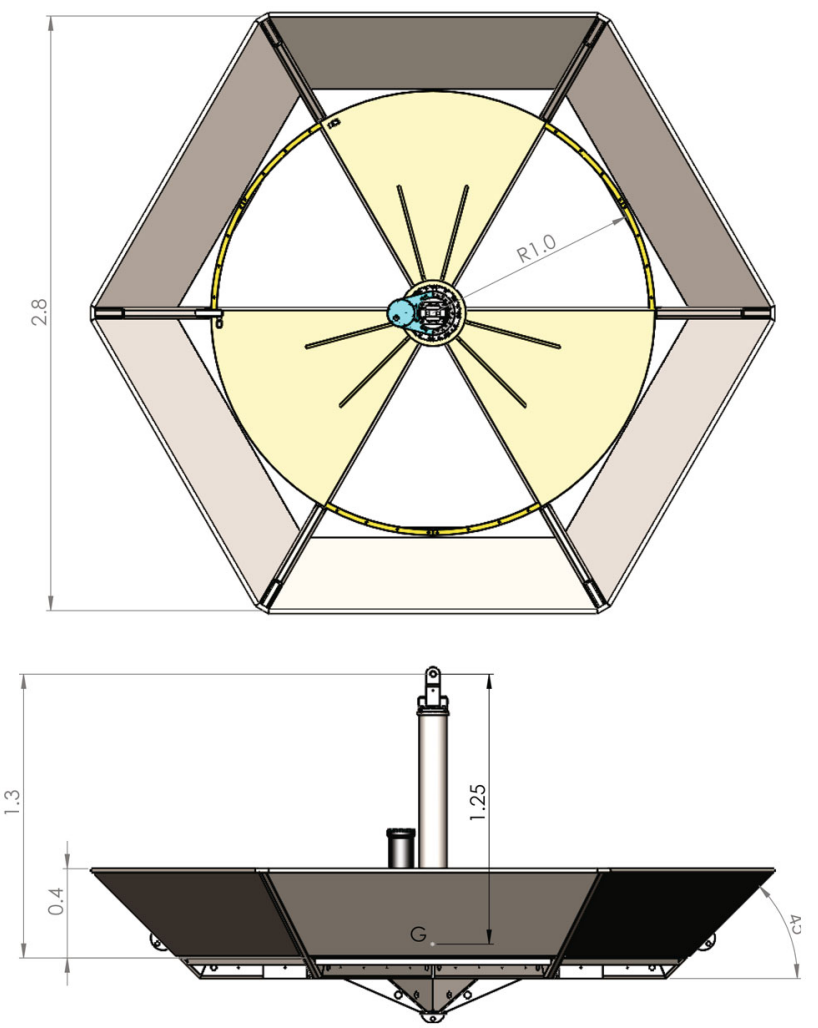

Fig. 5 MBARI-WEC heave cone layout drawing with top and side views. Length dimensions are in meters and angles are in degrees

the surface buoy as measured by an $89 \mathrm{kN}(20,000 \mathrm{lb})$ load cell. Also present are a gas make-up pump that can (slowly) move gas from one side of the piston to the other, a gas relief valve that can reverse that process, and a switched power supply that provides $48 \mathrm{~V}$ to the heave-cone.

The electro-hydraulic device consists of a piston driving oil through a fixed displacement hydraulic motor that is directly coupled to a three-phase brushless motor/generator. There is no hydraulic rectification and the motor reverses rotary direction with a change in piston direction of motion. This component contains a $10 \mathrm{~kW}$ (peak) four-quadrant power converter that controls the current in the motor windings to a set value. If the prescribed torque opposes the motion

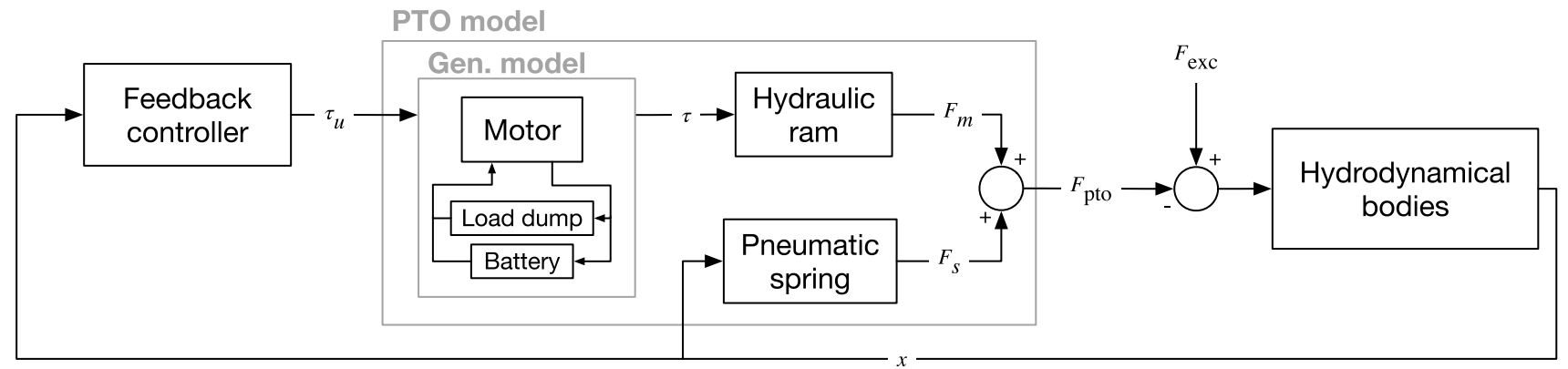

Fig. 6 PTO system electro-hydraulic block diagram 
of the motor, the system acts as a generator and boosts the voltage coming out of the three-phase generator to $325 \mathrm{~V}$ for transmission up to the battery pack on the buoy. This behaves as a current limited battery charger, if the batteries cannot accept the necessary power, the energy is directed instead to a submerged load-dump heater which is part of the PTO device. This load dump is sized to absorb all the power in case the batteries are full or disconnected.

If the commanded torque is in the same direction as the motion, the system acts as a motor and drives the ram with a force proportional to the commanded current. This draws energy out of the battery (up to a specified current limit). In addition to the current control duties, this micro-controller provides a range of telemetry to the buoy at $10-50 \mathrm{~Hz}$, this data includes motor RPM, motor winding current which indicates torque, bus voltage, load dump current, battery current (plus or minus) as well as a variety of controller status bits.

By default, this controller acts in a standalone "linear generator" mode, in which the generator exerts a torque that is linearly proportional to the motor RPM. In the event that communications from the surface are lost, the system reverts to this linearly proportional control default. Alternatively, the buoy control computer can command any torque it likes based on an algorithm running top-side. In this case, the power converter controller attempts to achieve the requested torque, but will self-limit in some situations for device safety considerations. Figure 6 also shows the relation between the motor/drive, the load dump, and the battery system.

The motor-drive power electronics in this system were custom developed for this application because the waveenergy problem at this scale has some specific requirements that are hard to achieve using commercially available power electronics. In particular, the efficiency requirements of the system are important, especially at low speed. In this type of motor drive, switching losses in the transistors are a significant component of the total losses and unfortunately do not diminish at low speeds and winding currents like other resistive losses in the system. For this reason, the motor drive developed for the MBARI-WEC turns off the transistor bridge at low speeds/torques to eliminate this loss. Additional features that are atypical of commercially available systems are also required because of the autonomous and remote use of the system. Under-voltage, over-speed, and all other conditions must be handled gracefully and allow the system to continue operating. In particular, the system must also bootstrap itself and turn on in the case of the batteries being disconnected, to ensure a load is always present in the system.

\section{Hydrodynamic modeling}

To enable future model-based system and control design, two hydrodynamic models have been developed: a frequency- domain model, which will provide a useful means of performing model based design (Sect. 3.1) and a time-domain model (Sect. 3.2) which provides a useful benchmark comparison point for the frequency-domain model.

\subsection{Frequency-domain model}

If we consider each of the two bodies within the device (the buoy and heave cone as described in Sect. 2) to be governed by an impedance model, we can write the equations of motion as follows.

$\hat{Z}\left(\omega_{l}\right) \hat{V}\left(\omega_{l}\right)=\hat{F}_{\text {ext }}\left(\omega_{l}\right)$

Here, $\hat{Z}\left(\omega_{l}\right) \in \mathbb{C}^{2 \times 2}$ is the complex impedance matrix and $l \in[1: n]$, where $n$ is the number of frequency components. The elements of the matrix $\hat{Z}\left(\omega_{l}\right)$ are

$$
\begin{aligned}
\hat{Z}_{j, k}\left(\omega_{l}\right)= & B_{j, k}\left(\omega_{l}\right)+b_{j, k} \\
& \quad+i\left(\omega_{l}\left(m_{j, k}+A_{j, k}\left(\omega_{l}\right)\right)-\frac{K_{j, k}}{\omega_{l}}\right) \\
= & R_{j, k}\left(\omega_{l}\right)+i X_{j, k}\left(\omega_{l}\right) .
\end{aligned}
$$

The subscripts $j$ and $k$ relate to the bodies that comprise the WEC (buoy: 1, heave cone: 2), such that, for example, $\hat{Z}_{1,2}(\omega)$ describes the impedance on the buoy due to motion of the heave cone. The components $R_{j, k}(\omega)$ and $X_{j, k}(\omega)$ are the resistance and reactance, respectively. The terms $B_{j, k}(\omega)$ and $b_{j, k}$ are the frequency-dependent linear damping ("radiation damping") and additional linear damping (accounting for both viscous friction in the water and mechanical friction), respectively. Likewise, $A_{j, k}(\omega)$ and $m_{j, k}$ are the added and rigid-body inertias, respectively. Finally, $K_{j, k}$ is the balance of stiffness terms. In addition, the presence of the pneumatic spring in parallel with the hydraulic PTO coupling the two bodies (see Fig. 6), and the presence of a mooring affecting the heave plate, the stiffness term is

$K=\left[\begin{array}{cc}K_{h s, 1}+K_{\text {spring }} & -K_{\text {spring }} \\ -K_{\text {spring }} & K_{h s, 2}+K_{\text {spring }}+K_{\text {moor }}\end{array}\right]$

where $K_{h s}$ is the hydrostatic stiffness. Similarly,

$b=\left[\begin{array}{cc}b_{\mathrm{drag}, 1} & 0 \\ 0 & b_{\mathrm{drag}, 2}+b_{\text {moor }}\end{array}\right]$

for the effect of the mooring line, $b_{\text {moor }}$. Values of $b_{\text {drag, } 1}$ and $b_{\text {drag,2 }}$ were based upon the iterative Lorentz linearization (Terra et al. 2005) of the quadratic drag coefficient in the heave direction for the buoy and heave plate (respectively) using the periodic PTO forcing time series.

The complex velocity of each body is described by $\hat{V}\left(\omega_{l}\right)=\left[\hat{V}_{1}\left(\omega_{l}\right), \hat{V}_{2}\left(\omega_{l}\right)\right]^{T}$. External forces on the system 
are given by $\hat{F}_{\text {ext }}=\left[\hat{F}_{\text {ext, } 1}\left(\omega_{l}\right), \hat{F}_{\text {ext,2 }}\left(\omega_{l}\right)\right]^{T}$. Each term of external force is itself composed of two components: the excitation force, $\hat{F}_{\text {exc }}$, and the PTO force, $\hat{F}_{\text {pto }}$.

$\hat{F}_{\text {ext }}=\hat{F}_{\text {exc }}-\hat{F}_{\text {pto }}$,

where the sign convention has the PTO force opposing the excitation (i.e., negative feedback) - this is also shown in Fig. 6. The PTO force acts in equal magnitude and opposite sign on bodies 1 and 2 , such that

$\hat{F}_{\text {pto }}=\left[\begin{array}{c}1 \\ -1\end{array}\right] \hat{f}_{\text {pto }}$

where $\hat{f}_{\text {pto }} \in \mathbb{C}^{1 \times n}$.

To find a simple means of specifying $\hat{f}_{\text {pto }}$, we can compress the system to a single degree of freedom by solving for the relative motion between the two bodies. First, returning to (1), we can write

$\hat{V}=\hat{Z}^{-1} \hat{F}_{\text {ext }}$.

To solve for the relative motion, we can further write

$$
\begin{aligned}
\Delta \hat{V} & =\hat{V}_{1}-\hat{V}_{2}=[1-1] \hat{V} \\
& =[1-1] \hat{Z}^{-1} \hat{F}_{\text {ext }} .
\end{aligned}
$$

Within this composite system, to consider the impedance observed at the PTO we can disregard other external forces (i.e., by setting $\hat{F}_{\text {exc }}=0$ ). Thus, from (8) we can find the composite system impedance.

$$
\begin{aligned}
\Delta \hat{V} & =-1[1-1] \hat{Z}^{-1} \hat{F}_{\text {pto }} \\
& =-1[1-1] \hat{Z}^{-1}\left[\begin{array}{c}
1 \\
-1
\end{array}\right] \hat{f}_{\text {pto }} \\
& =\hat{Z}_{\text {eq }}^{-1} \hat{f}_{\text {pto }}
\end{aligned}
$$

The final expression of (9) defines the relative motion of the two bodies in the system in terms of a single PTO force, $\hat{f}_{\text {pto }}$, and an equivalent admittance, $\hat{Y}_{\mathrm{eq}}=\hat{Z}_{\mathrm{eq}}^{-1}$. Some manipulation of (9) gives

$\hat{Y}_{\mathrm{eq}}=\hat{Z}_{\mathrm{eq}}^{-1}=\frac{\hat{Z}_{1,1}+\hat{Z}_{1,2}+\hat{Z}_{2,1}+\hat{Z}_{2,2}}{\hat{Z}_{1,2} \hat{Z}_{2,1}-\hat{Z}_{1,1} \hat{Z}_{2,2}}$.

With (10), we now have a succinct means of describing the response of the WEC system to any input. Returning to the task of specifying $\hat{f}_{\text {pto }}$, we can maximize mechanical power by setting the PTO force based on an impedance, as shown in Fig. 7. It is well known that to maximize power, one would

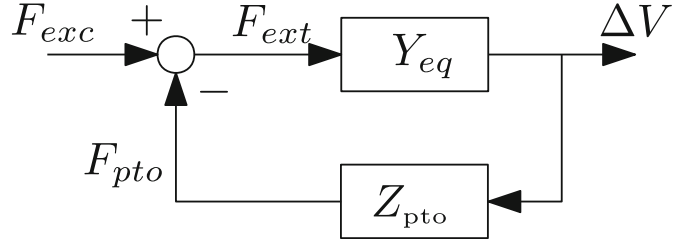

Fig. 7 Closed loop control of a WEC based on an impedance

set the PTO impedance based on the complex conjugate of the system impedance (see, e.g., Falnes 2002).

$$
\begin{aligned}
& \hat{f}_{\text {pto }}=\hat{Z}_{\text {pto }} \cdot \Delta \hat{V} \\
& \text { where } \hat{Z}_{\text {pto, opt }}=\hat{Z}_{\text {eq }}^{*} .
\end{aligned}
$$

However, as discussed in Bacelli and Coe (2020); Coe et al. (2020), a causal PTO impedance can be used to approximate the condition specified in (11) for some limited frequency range. Given the band-limited nature of ocean waves, the tuning of such a controller can be updated intermittently to track the slowly varying changes in sea state. This "self-tuning" approach was shown by Forbush et al. (2020).

While any number of feedback formulations can be applied, we can consider a proportional-integral (PI) controller here for the time being. In this case, we set

$\hat{Z}_{\mathrm{pto}}=k_{P}-i \frac{k_{I}}{\omega}$

Thus, we can maximize power as follows by tuning the controller gains $k_{P}$ and $k_{I}$.

$\arg \min _{\hat{Z}_{\mathrm{pto}}\left(k_{P}, k_{I}\right)} P\left[\hat{F}_{\mathrm{exc}}(S(\omega)), \hat{Z}_{\mathrm{eq}}, \hat{Z}_{\mathrm{pto}}\left(k_{P}, k_{I}\right)\right]$.

Here, the optimization problem has been defined to minimize work done by the WEC, such that negative work equates to absorbed power. Note that the solution to this problem is dependent on the prevailing sea state, $S(\omega)$, and the device dynamics, $\hat{Z}_{\text {eq }}$ and $\hat{F}_{\text {exc }}$.

\subsection{WEC-Sim models}

To provide a comparison point off which to judge the viability of using the impedance model described in Sect. 3.1, a weakly nonlinear time-domain model was developed in WEC-Sim (Ruehl et al. 2016). WEC-Sim uses a time-domain Cummins' formulation (Cummins 1962) to model WEC dynamics and can be constructed from boundary element method (BEM) data, and expanded to model nonlinearity in the physical system. The specific sources of nonlinear behavior considered herein are kinematics, mooring, and a quadratic drag model based upon buoy and heave plate geometry (Table 2). In the case of the asymmetric heave cone, a direction-specific drag 
Table 2 Quadratic $\left(C_{d}\right)$ drag coefficients and projected area $(A)$ used in WEC-Sim in [heave, surge, pitch], and linear heave damping $(B)$

\begin{tabular}{llll}
\hline & $C_{d}$ & $A\left[\mathrm{~m}^{2}\right]$ & $B[\mathrm{~N}-\mathrm{s} / \mathrm{m}]$ \\
\hline Buoy & {$[1,1.15,0.5]$} & {$[5.47,2.96,5.47]$} & 665.30 \\
Cone & {$[*, 0.8,1]$} & {$[6.56,1.12,6.56]$} & 965.53 \\
\hline
\end{tabular}

Table 3 Linearized mooring parameters

\begin{tabular}{lll}
\hline$K_{\text {moor }}[\mathrm{N} / \mathrm{m}]$ & $b_{\text {moor }}[\mathrm{N}-\mathrm{s} / \mathrm{m}]$ & $T[\mathrm{~N}]$ \\
\hline 6.47 & 81.14 & 233.22 \\
\hline
\end{tabular}

Table 4 WEC-Sim model configurations for comparison with impedance model

\begin{tabular}{llll}
\hline Model ID & DOF & Drag & Mooring \\
\hline WS1 & Heave only & Linear & Matrix \\
WS2 & Planar motion & Quadratic & Matrix \\
WS3 & Planar motion & Quadratic & MoorDyn
\end{tabular}

Note that WS0 is not shown, but is the same as WS1, but with a rigid tether

coefficient of 1.45 (moving upward) and 0.85 (moving downward) is used (as indicated by the asterisk in Table 2), the decoupling of the heave plate and buoy motion when the tether is slack, and the single-point catenary mooring are the modeled non-linearities.

Additionally, MoorDyn (Hall and Goupee 2015) can be used to model the mooring based solely upon physical component description and advise linear approximation (i.e., $K_{\text {moor }}$ ) in absence of test data. In this case, a heave-only WEC-Sim model using MoorDyn was run, and the linear mooring stiffness, damping, and pretension were found by solving a least-squares problem using the the forces and motions produced by the MoorDyn simulation. Because the suspended wet mass of the single-point catenary mooring will change based upon sea-level changes (i.e., tides) and the lateral and vertical displacement of the heave plate to the mooring anchor, this approximation is only anticipated to be valid in the vicinity of the linearized location (Table 3). However, excellent agreement was observed under these conditions $\left(r^{2}=0.99\right)$.

\section{Comparison of hydrodynamical models}

As the impedance model detailed in Sect. 3.1 will be used for control design, it is necessary to assess the accuracy of this model. Here, we compared the impedance model against a series of models developed in WEC-Sim. The different WEC-Sim model configurations are summarized in Table 4 and illustrated in Fig. 8. The WS1 model is a heave-only model with only linear dynamics. The WS2 model adds additional kinematics for planar motion (heave, surge, pitch) and quadratic drag. WS3 is a four-body model (float, PTO cylinder, tether, and heave cone), and is considered the closest approximation of the physical device used in this comparison.

By treating the WEC-Sim results as numerical experiments, system identification (SID) methods can be applied to produce linear impedance models (Bacelli et al. 2017; Pintelon and Schoukens 2012; Ljung 1999). In this case, the PTO was excited with a pink spectra from 0.015 to $1.5 \mathrm{~Hz}$. Although unconstrained in surge and pitch for WS2 and WS3, in the absence of waves or other sources of surge or pitch forces, the PTO remains vertical. Note that in WECSim configuration WS1 - WS3, the slack tether decoupling was modeled, but PTO force amplitudes were selected such that the tether remained taut throughout all device motion. Figure 9 shows a Bode plot with these admittance models, alongside the admittance model developed directly from the BEM coefficients per (10). The WEC-Sim results agree well with BEM estimates near the resonance peak of the heave cone single-body admittance, but the higher frequency resonance peak occurs at a slightly lower frequency than predicted from BEM, which itself agrees closely with the single-body buoy impedance.

While Fig. 9 conveys the matching of these models in linear terms, it is also necessary to understand how well the linear model captures the dynamics of the WEC (i.e., the amount of nonlinearity present in the system and not captured by a linear model). Figure 10 shows the magnitude of velocity response from each of the four models in a regular wave with $H=1.25 \mathrm{~m}, T=8 \mathrm{~s}$, which is considered somewhat representative of the wave climate at the Monterey Bay deployment site during typical summer deployments. ${ }^{1}$ An optimal linear damping of $851 \mathrm{Ns} / \mathrm{m}$ was applied via the PTO (Falnes 2002; Coe et al. 2020). Note that, per Fig. 9 and Table 1 , this $8 \mathrm{~s}$ wave period is nearly the resonant frequency of the heave plate, and near the largest resonance peak of the equivalent impedance As a result, this case shows large-amplitude relative body motion, resulting in significant tether slack not captured in linear frequency-domain models (see time history in Fig. 11). For an additional point of comparison, a fourth WEC-Sim configuration (WS0) was tested in which a rigid tether was modeled. Otherwise identical to WS1, WS0 performs equivalently when no tether slack occurs.

A contrasting sea-state, with $H=1 \mathrm{~m}, T=5.5 \mathrm{~s}$, not expected to cause tether slack was also simulated. In this

\footnotetext{
1 Note that a regular wave is used here instead of an irregular wave to enable a more clear analysis of nonlinearities. However, in a real sea state the energy would be more broadly distributed across a range of frequencies.
} 


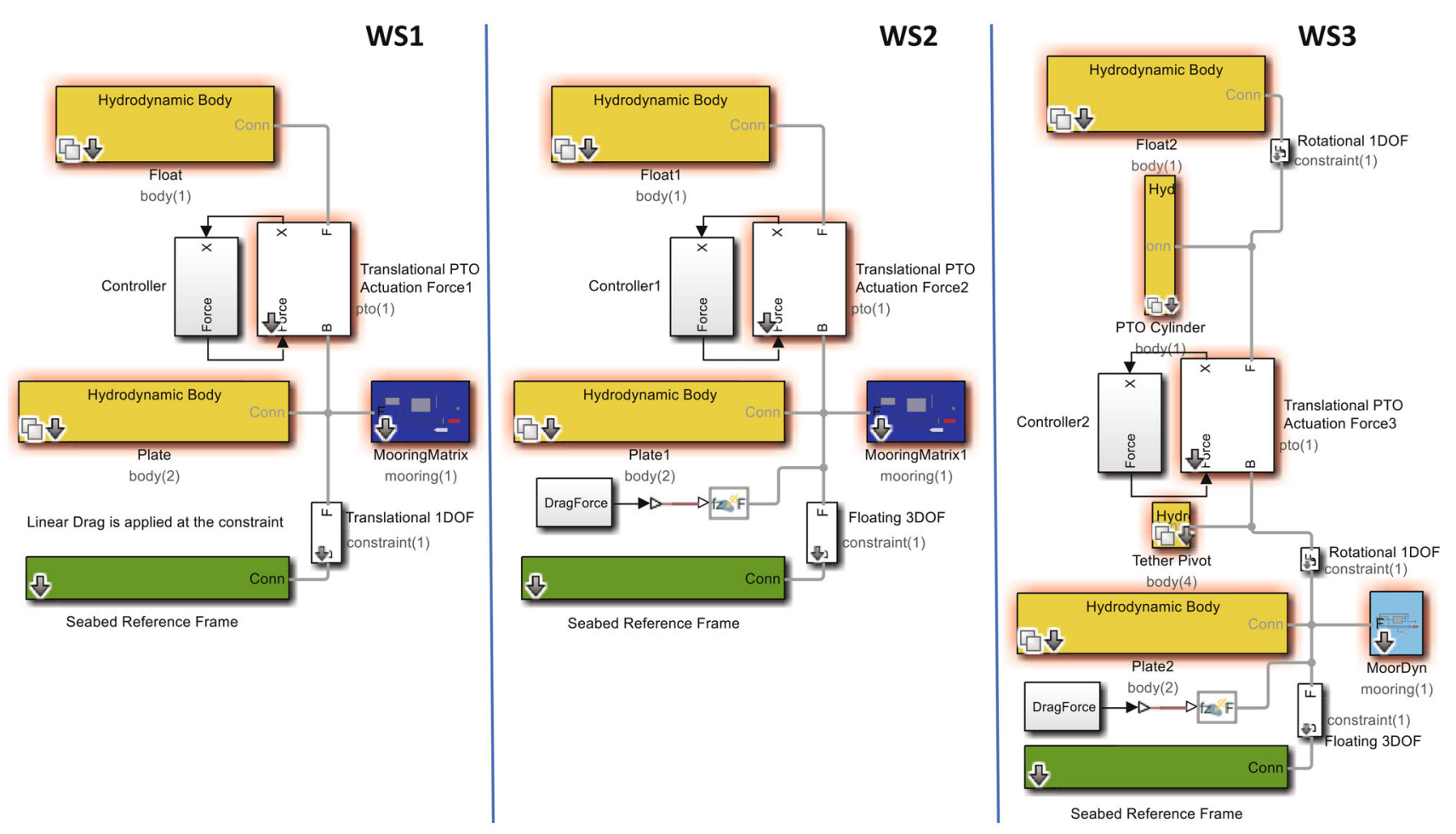

Fig. 8 Screenshots of WEC-Sim models in MATLAB Simulink showing different model configurations. See Table 4 for further explanation. Note that WS0 is not shown, but is the same as WS1, but with a rigid tether
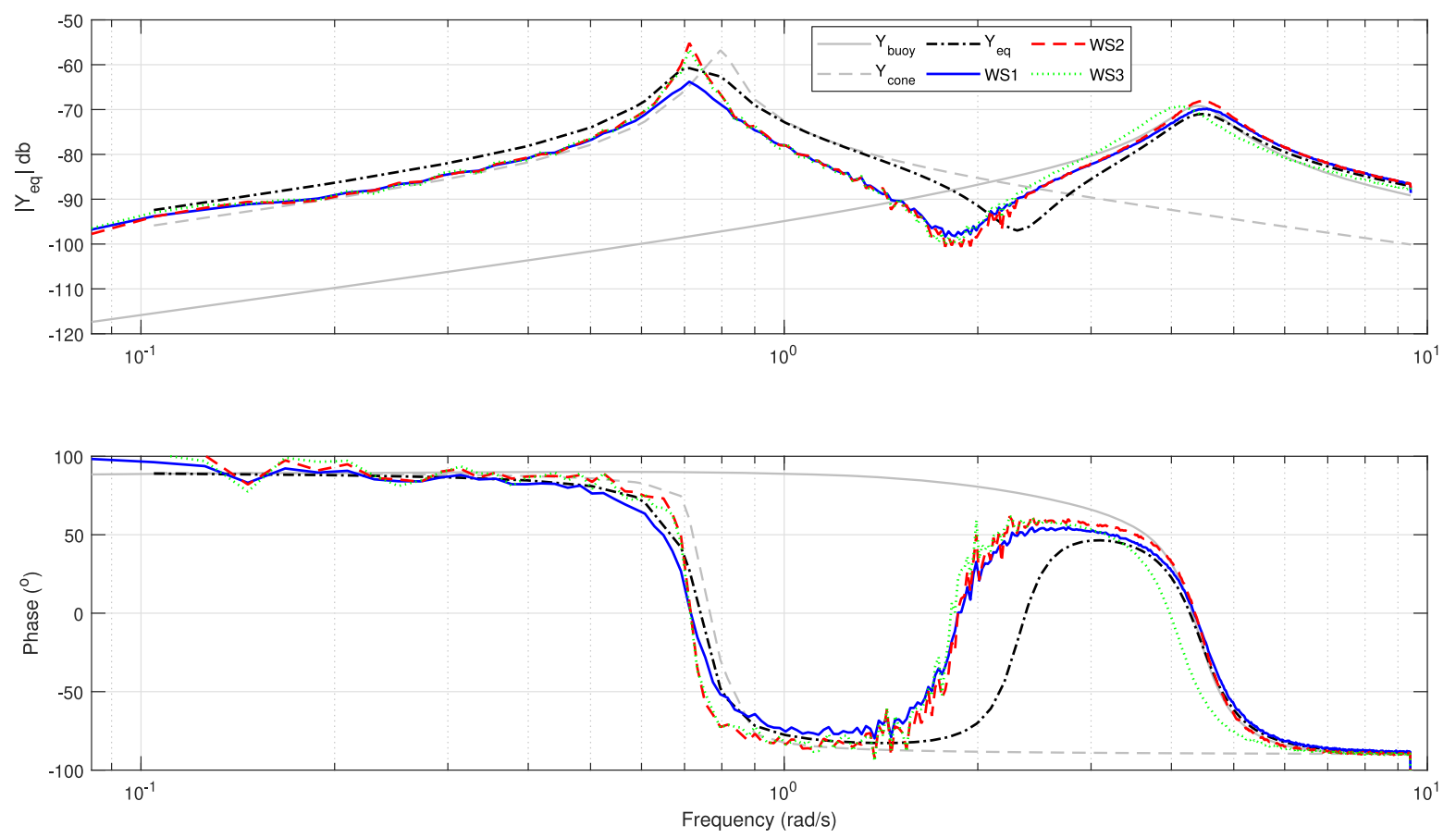

Fig. 9 Comparison of numerical modeling results: Bode plot of admittance calculated from WEC-Sim models compared against system admittance directly from BEM coefficients. Single-body buoy and heave plate admittance are also shown. Impedance model: $Y_{\text {eq }}$, WEC-Sim models: WS\# (Table 4 and Fig. 8 for configurations) 


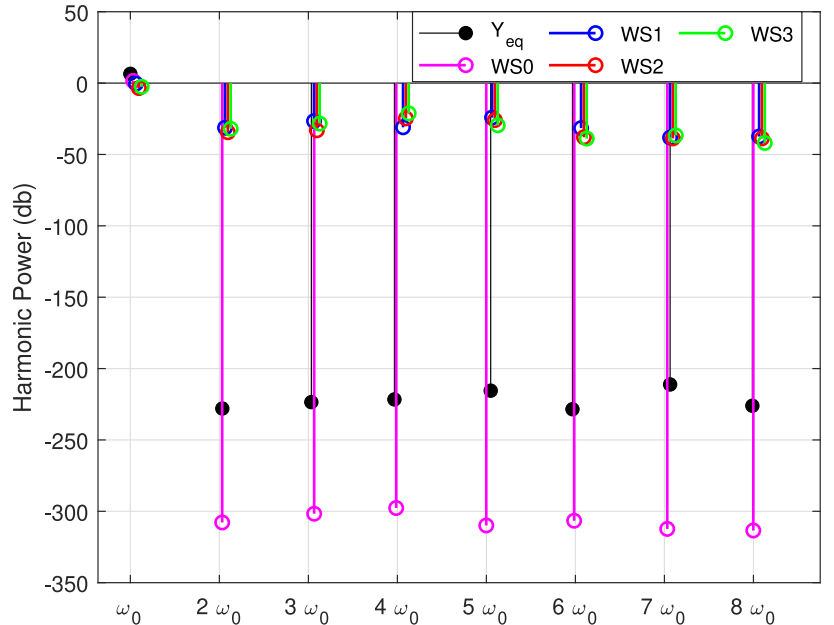

Fig. 10 Comparison of numerical modeling results: magnitude and phase response of models for a regular wave with $H=1.25 \mathrm{~m}, T=8 \mathrm{~s}$. All cases are evaluated at resonances of the excited frequency, minor offsets are introduced in the plot to avoid overlapping similar data. Impedance model: $Y_{\text {eq }}$, WEC-Sim models: WS\# (Table 4 and Fig. 8 for configurations)
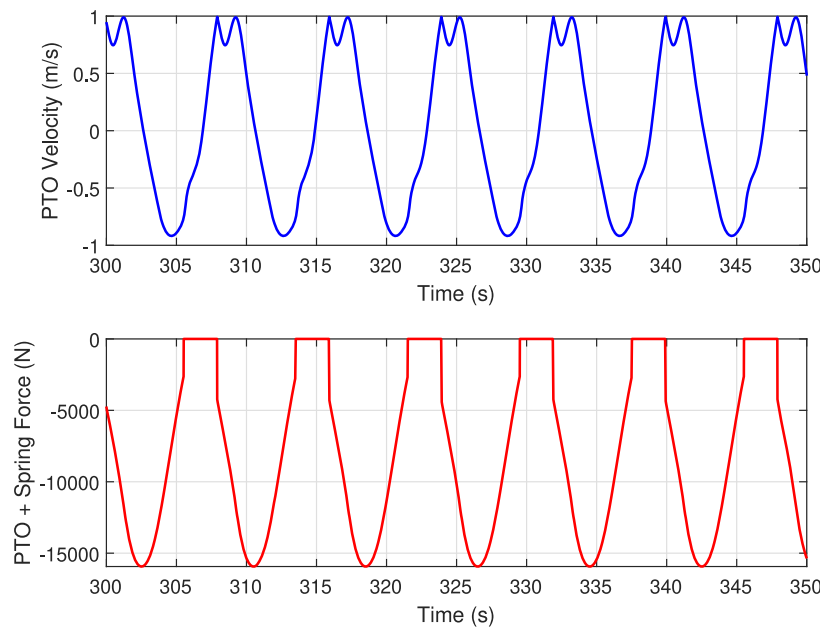

Fig. 11 PTO and spring force with PTO velocity for WS2 in $T=8$, $H=2.5$. The zeros of PTO and spring force indicate tether slack events

case the optimal damping factor for the PTO is $943 \mathrm{Ns} / \mathrm{m}$. The results of this case are shown in Fig. 12,

From Figs. 10 and 12, we can see the level of nonlinear content present in each model by the magnitude of the excited harmonics. The linear model, $\hat{Y_{\text {eq }}}$ contains effectively no energy at higher harmonics, but is presented here so the extent of numerical error can be evaluated. In the larger seastate (Fig. 10), the substantial differences between WS0 and WS1 suggest that tether slack is the most significant source of nonlinearity; this can be confirmed by Fig. 11. Further, note that substantially less energy is contained at higher harmonics for WS1-WS3 in the smaller sea state (Fig. 12), again highlighting the significant effect of tether slack. In

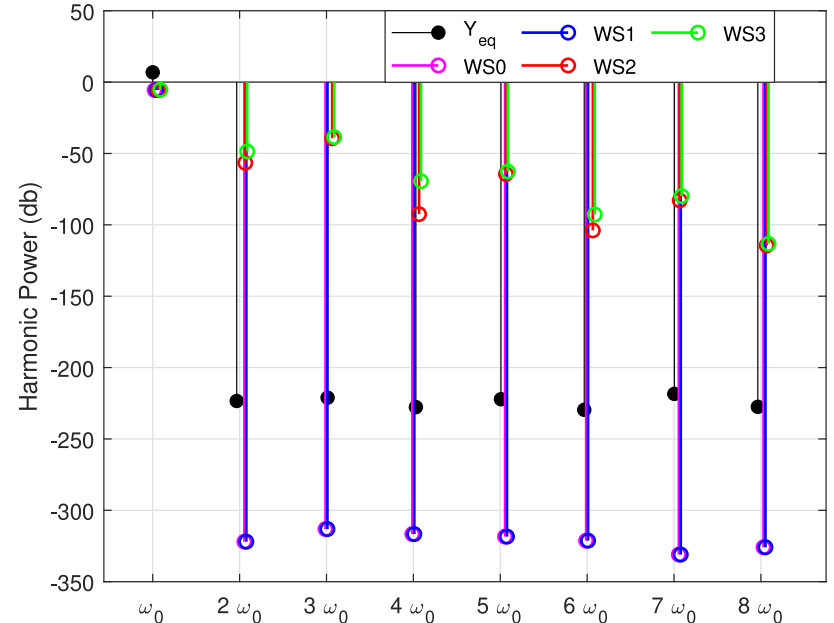

Fig. 12 Comparison of numerical modeling results: magnitude and phase response of models for a regular wave with $H=1 \mathrm{~m}, T=5.5 \mathrm{~s}$. All cases are evaluated at resonances of the excited frequency, minor offsets are introduced in the plot to avoid overlapping similar data. Impedance model: $Y_{\text {eq }}$, WEC-Sim models: WS\# (see Table 4 and Fig. 8 for configurations)

Table 5 Comparison of numerical modeling results: percent total harmonic distortion for regular wave simulations Impedance model: $Y_{\mathrm{eq}}$, WEC-Sim models: WS\# (see Table 4 and Fig. 8 for configurations)

\begin{tabular}{llllll}
\hline Wave state & $Y_{\text {eq }}$ & WS0 & WS1 & WS2 & WS3 \\
\hline$T=8 \mathrm{~s}, H=1.25 \mathrm{~m}$ & 0.0 & 0.0 & 9.8 & 12.7 & 14.3 \\
$T=5.5 \mathrm{~s}, H=1 \mathrm{~m}$ & 0.0 & 0.0 & 0.0 & 2.1 & 2.3 \\
\hline
\end{tabular}

the smaller sea-state, where there is no tether slack, WS2 and WS3 demonstrate that the impact of quadratic drag and nonlinear mooring: energy at each higher harmonic increases monotonically from WS1 (identical to WS0) to WS3. There is not a substantial difference in the higher harmonic content between WS1 and WS2-3 in the larger sea state. This implies that the addition of a surge/pitch degree of freedom, which one might expect to present disagreements with a model derived with a vertical PTO, does not have a significantly non-linear effect at the investigated wave amplitudes.

Quantitatively, we can also find the total harmonic distortion (THD) from each velocity signal (see Table 5). Again, the linear BEM solution has a theoretical THD of zero, but is included here to quantify the extent of numerical error. Aligning with intuition, the progressive introduction of nonlinearities to simulation cases serves to increase the THD monotonically from WS0 to WS3, with the most striking separation occurring between WS0 and WS1, indicating that tether slack is the primary nonlinearity. Further, there is a striking reduction in THD for all cases in the smaller seastate. However, it is notable that THD remains fairly low in all cases, suggesting that nonlinear will not strongly limit the utility of strictly linear models for controller development. 


\section{Discussion}

Generally, there is strong agreement in the identified systems observed for all WEC-Sim realizations (Fig. 9). Additionally, the nonlinearity in the WEC-Sim models are relatively small (see Fig. 12 and Table 5). This suggests that, at least for the excitation used here, nonlinearities have a minor impact, and in turn, that the linear impedance system model adequately describes complex system dynamics for the purposes of controller design. This is especially true if one considers that power is proportional to velocity squared.

It is interesting to note the disagreement between the admittance model and WS1, as shown Fig. 9. One potential source for this discrepancy is the infinite-frequency added mass $\left(A_{\infty}=\lim _{\omega \rightarrow \infty} A(\omega)\right)$. WEC-Sim relies fundamentally on the time-domain Cummins' formulation, which uses an estimate of infinite-frequency added mass, along with impulse response functions, to determine WEC dynamics at each time-step via a convolution integral (see, e.g., (Tom et al. 2015)). Ideally, a boundary element method code can estimate the infinite frequency added mass, however in this case, such an estimate was not available, and so the values of added mass at the highest frequency, which were approaching, though not yet fully converged to an asymptote, were used instead. While this is expected to be a reasonable approximation, some of the distinctions in Fig. 9 are the apparent consequence.

Varying levels of nonlinearity in the two regular wave test cases (see Table 5) highlight the amplitude-dependency of system nonlinearity: the linear impedance model may be an adequate descriptor for small perturbations, but large sea-states may cause substantial deviation. However, in this particular case, tether slack is clearly undesirable from an operational perspective. Thus, a properly-designed controller may itself confine operations to a more-nearly linear regime. Also undesirable, a significant pitch displacement of the PTO cylinder may reduce the accuracy of the identified system model, which was identified with a nominally vertical PTO. However, given the depth of the heave cone during typical operation, this latter case would represent a very extreme operating condition when one would naturally not expect a highly accurate linear model. Avoiding significant nonlinear behavior may not be ideal or possible for all WEC and PTO archetypes, and the limitations of a linear model should be considered in the context of each specific device.

\section{Conclusion}

The MBARI-WEC is a first of its kind device with an extended deployment history. The device has been designed for support of autonomous sensors and to eventually support AUV charging. With roughly ten years worth of development and deployment experience, the MBARI-WEC has a plethora of learning experiences to offer.

This study has presented the particulars of the MBARIWEC design for general reference and to support future open access to the MBARI-WEC. Additionally, frequency domain hydrodynamic model was derived and compared with similar models solved in the time domain via WEC-Sim. The comparison of these models shows that, in comparison to the higher fidelity WEC-Sim model, the frequency domain model does a good job of capturing the device dynamics. Nonlinearities seen in the WEC-Sim results tended to be small, especially when slack line events that would be avoided during actual operation did not occur. These findings support the application of the frequency domain model for future co-design efforts to improve the performance of the MBARI-WEC.

Efforts are currently underway to establish the MBARIWEC as an open platform which can be accessed by the general research community and leveraged for various purposes. Future efforts will include an in-depth analysis of the MBARI-WEC PTO, with experimental and numerical modeling. Additionally, measurements from wave buoys and hindcast data will be used in combination with device telemetry to further validate the hydrodynamic models proposed in this paper. These analyses will support design improvements to the system. One potential point of interest will be the investigation of ways in which the WEC system and control design can be modified to improve performance, specifically to reduce loading in large sea states and improve power generation in smaller sea states. MBARI's experience with this system to date indicates that an important improvement to pursue is to make the system smaller, as a 10-20\% reduction in size would simplify deployment and use significantly. Currently, no advanced control systems have been implemented for this system, so this is an important area of research that can help meet the size goals of the system without sacrificing the energy captured.

While the comparison of numerical models presented in this paper do give some justification for leveraging the equivalent impedance model for future system and control design efforts, the model has not been validated against experimental data. During previous recent deployments of the MBARIWEC, no wave data in the vicinity of the device has been available. ${ }^{2}$ Thus, the decision was made in this study to focus on the comparison of numerical models. However, there are plans to deploy wave buoys and use spectral hindcast models to support a comparison between experimental measurements and numerical models in the future.

\footnotetext{
${ }^{2}$ Earlier in the deployment history of the MBARI-WEC (when the device design was slightly different), CDIP did maintain a buoy in the area (CDIP156: https://cdip.ucsd.edu/themes/cdip?pb=1\&u2=s:156:st: $1 \& \mathrm{~d} 2=\mathrm{p} 9)$, but this buoy has since been decommissioned.
} 
Acknowledgements The authors would like to acknowledge funding support from the US Department of Energy's Water Power Technologies Office. Sandia National Laboratories is a multi-mission laboratory managed and operated by National Technology and Engineering Solutions of Sandia, LLC., a wholly owned subsidiary of Honeywell International, Inc., for the U.S. Department of Energy's National Nuclear Security Administration under contract DE-NA0003525. This paper describes objective technical results and analysis. Any subjective views or opinions that might be expressed in the paper do not necessarily represent the views of the U.S. Department of Energy or the United States Government; Office of Energy Efficiency and Renewable Energy.

\section{Declarations}

Conflict of interest The authors declare that they have no conflict of interest.

Open Access This article is licensed under a Creative Commons Attribution 4.0 International License, which permits use, sharing, adaptation, distribution and reproduction in any medium or format, as long as you give appropriate credit to the original author(s) and the source, provide a link to the Creative Commons licence, and indicate if changes were made. The images or other third party material in this article are included in the article's Creative Commons licence, unless indicated otherwise in a credit line to the material. If material is not included in the article's Creative Commons licence and your intended use is not permitted by statutory regulation or exceeds the permitted use, you will need to obtain permission directly from the copyright holder. To view a copy of this licence, visit http://creativecomm ons.org/licenses/by/4.0/.

\section{Appendix: System telemetry}

Table 6 provides a listing of system telemetry as of the publication of this paper.

Table 6 MBARI-WEC system telemetry

\begin{tabular}{ll}
\hline Signal name & Description \\
\hline Battery Controller (BC) & \\
BC_Time & Unix time of each data point \\
BC_Voltage & Main battery voltage \\
BC_GroundFault & 0-20 mA output from Bender ground fault \\
& detector \\
BC_Ips & Current flowing to the 300 V-24 V power \\
& supply for Linux buoy controller and \\
& accessories. \\
BC_VStopCharge & Voltage indicating number of 12 V \\
& batteries that are at or above the \\
specified "StopCharge" voltage & \% of LEL of H2 in battery box \\
BC_Hydrogen & A variety of status bits indicating the state \\
BC_Status & of the battery system \\
A status voltage that indicates how many & cells are currently bypassing in \\
balancing mode.
\end{tabular}

Table 6 continued

\begin{tabular}{ll}
\hline Signal name & Description \\
\hline BC_VStopCharge & $\begin{array}{c}\text { A status voltage that indicates how many } \\
\text { cells are over-voltage, this tells the } \\
\text { power converter to back off the voltage } \\
\text { Battery pack voltage }\end{array}$ \\
BC_Voltage & 4-20 mA signal from Bender ground fault \\
BC_GroundFault & detector indicating ground faults on \\
& 300 bus
\end{tabular}

Power Converter (PC)

\begin{tabular}{|c|c|}
\hline PC_Time & Unix time of each data point \\
\hline PC_BattCurr & Current flowing to or from batteries \\
\hline PC_Voltage & Bus voltage at power converter \\
\hline PC_RPM & $\begin{array}{l}\text { Motor shaft speed (positive RPM } \\
\text { corresponds to PTO ram retracting) }\end{array}$ \\
\hline PC_WindingCurrent & $\begin{array}{l}\text { Winding Quadrature Current, linearly } \\
\text { related to torque }\end{array}$ \\
\hline PC_LoadCurr & Current flowing to the load dump \\
\hline PC_Status & $\begin{array}{l}\text { A variety of status bits indicating state of } \\
\text { the power converter }\end{array}$ \\
\hline PC_DiffPress & $\begin{array}{l}0-10 \mathrm{psi}(0-0.69 \text { bar }) \text { signal indicating } \\
\text { hydraulic compensator pressure above } \\
\text { ambient }\end{array}$ \\
\hline PC_Scale & $\begin{array}{l}\text { User specified scale factor }(0.5: 1.2) \\
\text { applied to internally programmed } \\
\text { torque/speed curve }\end{array}$ \\
\hline PC_Retract & $\begin{array}{l}\text { User specified factor }(0.5: 1.2) \text { applied to } \\
\text { internally programmed torque/speed } \\
\text { curve during retraction only }\end{array}$ \\
\hline
\end{tabular}

Spring Controller (SC)

$$
\begin{aligned}
& \text { SC_Time } \\
& \text { SC_LoadCell }
\end{aligned}
$$

SC_LowerPressure

SC_UpperPressure

SC_Range

Unix time of each data point

0-20000 lb (0-89 kN) load cell reading, between PTO and buoy

Lower spring chamber pressure (psia)

Upper spring chamber pressure (psia)

PTO Ram position in inches (0: fully retracted; 80: fully extended).

SC_Status

A variety of status bits indicating state of the spring controller

Heave Cone Controller (TC)

\begin{tabular}{ll} 
TC_Time & Unix time of each data point \\
TC_ACcel1 & Heave Cone $x$-acceleration $\left(\mathrm{m} / \mathrm{s}^{2}\right)$ \\
TC_ACCel1 & Heave Cone $y$-acceleration $\left(\mathrm{m} / \mathrm{s}^{2}\right)$ \\
TC_ACCel1 & Heave Cone $z$-acceleration $\left(\mathrm{m} / \mathrm{s}^{2}\right)$ \\
TC_Qtn1 & Heave cone orientation quaternion \\
TC_Qtn2 & Heave cone orientation quaternion \\
TC_Qtn3 & Heave cone orientation quaternion \\
TC_Qtn4 & Heave cone orientation quaternion \\
TC_Mag1 & Heave cone magnetometer component 1 \\
TC_Mag2 & Heave cone magnetometer component 2 \\
TC_Mag3 & Heave cone magnetometer component 3 \\
SC_Status & Heave cone trefoil position and status \\
\hline
\end{tabular}


Table 6 continued

\begin{tabular}{ll}
\hline Signal name & Description \\
\hline Buoy GPS/IMU $(\mathrm{XB})$ & \\
XB_Time & Unix time of each data point \\
XB_Alt & Buoy vertical position \\
XB_Lat & Latitude from GPS \\
XB_Lon & Longitude from GPS \\
XB_NorthVel & North buoy velocity $(\mathrm{m} / \mathrm{s})$ \\
XB_EastVel & East buoy velocity $(\mathrm{m} / \mathrm{s})$ \\
XB_DownVel & Vertical buoy velocity $(\mathrm{m} / \mathrm{s})$ \\
XB_YawAng & Buoy yaw angle $(\mathrm{rad})$ \\
XB_PitchAng & Buoy pitch angle $(\mathrm{rad})$ \\
XB_RollAng & Buoy roll angle $(\mathrm{rad})$ \\
\hline
\end{tabular}

\section{References}

Bacelli G, Coe RG (2020) Comments on control of wave energy converters. In: IEEE Transaction on Control System Technologies pp 1-4, https://doi.org/10.1109/TCST.2020.2965916, https:// ieeexplore.iee.. rg/document/9005201

Bacelli G, Coe RG, Patterson D, Wilson D (2017) System identification of a heaving point absorber: Design of experiment and device modeling. Energies 10(10):472 https://doi.org/10.3390/en10040472, http://www.mdpi.com/1996-1073/10/4/472

Cavagnaro RJ, Copping AE, Green R, Greene D, Jenne S, Rose D, Overhus D (2020) Powering the blue economy: progress exploring marine renewable energy integration with ocean observations. Mar Technol Soc J 54(6):114-125

Coe RG, Bacelli G, Forbush D (2021) A practical approach to wave energy modeling and control. Renewable and Sustainable Energy Reviews 142:110791. https://doi.org/10.1016/j.rser.2021.110791

Copping A, LiVecchi A, Spence H, Gorton A, Jenne S, Preus R, Gill G, Robichaud R, Gore S (2018) Maritime renewable energy markets: power from the sea. Mar Technol Soc J 52(5):99-109

Cummins WE (1962) The impulse response function and ship motions. Tech. Rep. DTNSDRC 1661, Department of the Navy, David Taylor Model Basin, Bethesda. http://dome.mit.edu/handle/1721.3/ 49049

Driscol BP, Gish LA, Coe RG (2020) A scoping study to determine the location-specific wec threshold size for wave-powered auv recharging. IEEE J Ocean Eng. https://doi.org/10.1109/JOE.2020. 2973032, https://ieeexplore.ieee.org/document/9044418

Falnes J (2002) Ocean waves and oscillating systems. Cambridge University Press, Cambridge New York

Falnes J, Hals J (2012) Heaving buoys, point absorbers and arrays. Philos Trans R Soc A Math Phys Eng Sci 370(1959):246277. https://doi.org/10.1098/rsta.2011.0249, http://rsta. royalsocietypublishing.org/content/370/1959/246.abstract, http://rsta.royalsocietypublishing.org/content/370/1959/246. full.pdf + html

Forbush DD, Bacelli G, Spencer SJ, Coe RG (2020) A self-tuning WEC controller for changing sea states (in press). In: Proceedings of the 21st IFAC World Congress, vol 53, pp 12307-12312. https://doi. org/10.1016/j.ifacol.2020.12.1185, 21th IFAC World Congress

Green R, Copping A, Cavagnaro RJ, Rose D, Overhus D, Jenne D (2019) Enabling power at sea: opportunities for expanded ocean observations through marine renewable energy integration. In: OCEANS 2019 MTS/IEEE SEATTLE, IEEE, Seattle, WA, USA, pp 1-7, https://doi.org/10.23919/OCEANS40490.2019.8962706, https://ieeexplore.iee.org/document/8962706

Hagerman G (2002) Wave energy systems for recharging AUV energy supplies. In: Proceedings of the 2002 Workshop on Autonomous Underwater Vehicles, 2002., IEEE, San Antonio, TX, USA, pp 75-84, https://doi.org/10.1109/AUV.2002.1177207, https:// ieeexplore.iee.org/document/1177207

Hall M, Goupee A (2015) Validation of a lumped-mass mooring line model with DeepCwind semisubmersible model test data. Ocean Eng 104:590-603 https://doi.org/10.1016/j.oceaneng. 2015.05.035, https://www.sciencedirect.com/science/article/pii/ S0029801815002279

LiVecchi A, Copping A, Jenne D, Gorton A, Preus R, Gill G, Robichaud R, Green R, Geerlofs S, Gore S, Hume D (2019) Powering the blue economy; exploring opportunities for marine renewable energy in maritime markets. In: Tech. rep., US Department of Energy, Office of Energy Efficiency and Renewable Energy. Washington, DC, Washington, DC, https://www.energy.gov/sites/prod/ files/2019/03/f61/73355.pdf

Ljung L (1999) System identification-theory for the user. PrenticeHall

Pintelon R, Schoukens J (2012) System identification: a frequency domain approach. Wiley

Ruehl K, Michelen C, Bosma B, Yu YH (2016) WEC-Sim phase 1 validation testing: Numerical modeling of experiments. In: Proceedings of the ASME 2016 35th International Conference on Ocean, Offshore and Arctic Engineering (OMAE), American Society of Mechanical Engineers, ASME, Busan, South Korea, pp V006T09A026-V006T09A026. https://doi.org/ 10.1115/OMAE2016-54986

Terra GM, van de Berg WJ, Maas LR (2005) Experimental verification of Lorentz' linearization procedure for quadratic friction. Fluid Dyn Res 36(3):175. https://doi.org/10.1016/j.fluiddyn.2005. 01.005

Tom N, Lawson M, Yu YH(2015) Demonstration of the recent additions in modeling capabilities for the WEC-Sim wave energy converter design tool. In: Proceedings of the ASME 2015 34th International Conference on Ocean, Offshore and Arctic Engineering (OMAE), American Society of Mechanical Engineers, ASME, St. John's, Newfoundland, Canada, vol 56574, p V009T09A035, https://doi. org/10.1115/OMAE2015-42265

Publisher's Note Springer Nature remains neutral with regard to jurisdictional claims in published maps and institutional affiliations. 